Sociologie et sociétés

\title{
La question sociale
}

\section{Réfexion en marge d'un ouvrage de Pierre Rosanvallon}

\section{The Social Question. Reflections on a Work by Pierre} Rosanvallon

\section{Pierre DANDURAND}

Volume 28, numéro 2, automne 1996

Technologies médicales et changement de valeurs

URI : https://id.erudit.org/iderudit/001161ar

DOI : https://doi.org/10.7202/001161ar

Aller au sommaire du numéro

\section{Éditeur(s)}

Les Presses de l'Université de Montréal

\section{ISSN}

0038-030X (imprimé)

1492-1375 (numérique)

Découvrir la revue

Citer cet article

DANDURAND, P. (1996). La question sociale : réfexion en marge d'un ouvrage de Pierre Rosanvallon. Sociologie et sociétés, 28(2), 189-198.

https://doi.org/10.7202/001161ar

\section{Résumé de l'article}

En marge d'un ouvrage de Pierre Rosanvallon, l'auteur livre ses réflexions sur certaines des transformations actuelles de l'État-providence. Il conteste le fait que la forme assurancielle soit un mode " périmé " de régulation des solidarités ; il commente les " nouveaux droits sociaux " régissant les rapports État-prestataire ; il exprime de forts doutes sur la possibilité d'implanter une " société d'insertion " et sur l'interprétation voulant que l'État-providence ait maintenant à dispenser ses services à une " société d'individus ". 


\section{La question sociale Réflexions en marge d'un ouvrage de Pierre Rosanvallon*}

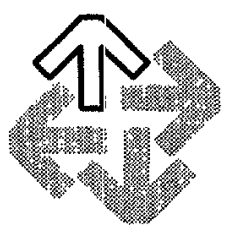

PIERRE DANDURAND

Dans notre appréhension de la société d'aujourd'hui, on est le plus souvent confronté soit à des analyses à tendance économiste, soit à des discours sur la postmodernité axés sur la culture et l'individu. Dans le premier cas, il n'est question que de restructuration de l'économie et de sa mondialisation comme les grands déterminants de cette fin de siècle. On parle de dualisation du marché du travail pour expliquer les divisions sociales, de crise fiscale pour traiter des problèmes de gestion étatique, des industries culturelles pour aborder la problématique des cultures, des problèmes d'employabilité pour traiter des chômeurs, des assistés sociaux, des familles monoparentales, et enfin on définit l'éducation selon les paramètres du capital humaỉn et des rapports formation/emploi. Le discours économique et la « science » économique, d'orientation libérale et néolibérale, déteignent fortement sur les analyses sociales et politiques quand ils ne prennent pas toute la place.

À l'autre extrême, il existe une abondante littérature sur les caractéristiques d'une société à modernité avancée qui s'implanterait sous nos yeux. Cette société se présenterait comme un ensemble extrêmement complexe d'entrelacs de relations interindividuelles, elle serait composée de personnes engagées dans des trajectoires individuelles et partagées entre de multiples identités sociales et culturelles. C'est à la limite une société d'individus, donc une société éclatée, quasi insaisissable, et où à toutes fins utiles la sociologie n'aurait plus vraiment d'objet. En sommie, à un pôle comme à l'autre, la dimension collective, l'épaisseur sociale du monde dans lequel nous vivons est fortement laminée.

Le récent ouvrage de Pierre Rosanvallon, La Nouvelle Question sociale. Repenser l'Étatprovidence (1995), rejoint par moments les thèses postmodernistes. Néanmoins, il a le mérite de pciser au centre de sa réflexion la question sociale, de s'arrêter aux modalités des liens sociaux, d'examiner l'état des solidarités dans le contexte de la crise de l'État-providence et du contrat qui lie les individus et les groupes dans les espaces nationaux. Les interrogations de l'auteur rejoignent celles de plusieurs chercheurs qui tentent de problématiser ce que sont nos

* Note de la direction. Nous tenons à remercier Renée B. Dandurand, qui a bien voulu confier à la revue ce texte cle Pierre Dandurand, écrit au cours du printemps et de l'été 1995. Notre collègue n'a pas pu en relire la dernière version et il n'a pas eu le temps de rédiger les notes dont la place était prévue dans le manuscrit. Sans doute aurait-il ajouté une brève conclusion. Nous avons retouché le manuscrit sur des points mineurs de forme et de style. La bibliographie regroupe des textes que l'auteur a vraisemblablement consultés au cours de son travail. 
sociétés, ce qu'elles sont en voie de devenir et l'orientation qu'elles doivent prendre. On est ainsi amené à un réexamen de dimensions majeures de la question sociale et à une saisie de processus nouveaux qui dorénavant régleraient en profondeur les liens sociaux.

\section{LA FORME ASSURANCIELLE, MODE PÉRIMÉ DE RÉGULATION DES SOLIDARITÉS ?}

L'évolution historique de l'État-providence s'est développée sur la base d'un système assuranciel. C'est ce que Rosanvallon s'attachera à montrer en soulignant l'importance qu'a eue la technologie sociale des assurances et la centralité d'une notion qui lui est indissociable, le risque, avant d'illustrer comment ces bases des régimes de protection sociale se sont érodées ces dernières années. Bien que son argumentation mette bien en évidence des aspects tout à fait cruciaux des transformations récentes des politiques sociales, il reste qu'elle comporte des lacunes qu'on ne peut passer sous silence. Tout particulièrement, elle ne réussit pas à dégager certains enjeux majeurs dans le champ du social et elle n'évoque qu'en sourdine comment ces enjeux sont liés aux positions et aux actions mobilisatrices d'agents sociaux qui, en définitive, contribuent à la structuration du social. En outre, la forme assurancielle ne recouvre qu'une partie des mesures de bien-être et de sécurité du revenu : privilégier la forme assurancielle de la protection sociale relègue en effet dans l'ombre les importants dispositifs d'assistance qui historiquement tendent à évoluer en constants rapports avec le volet assuranciel et se présentent comme l'un des invariants dans la structuration du champ des protections sociales.

Suivons d'abord l'auteur sur son propre terrain avant d'appliquer son analyse à quelques exemples puisés dans l'évolution de l'État-providence canadien et québécois.

La technique des assurances, qui permet une évaluation et un partage des risques jugés équitables, en serait venue à constituer la forme dominante imprimée aux liens de solidarité dans la société moderne. Mais voilà, cette forme instituée de la solidarité se révélerait maintenant dépassée : le mode assuranciel perd de sa pertinence et ne réussit plus à organiser et gérer les liens d'échange. Pourquoi ? Trois raisons auraient transformé la nature du risque socialement défini et les conditions de son partage, tout particulièrement depuis le début des années 1980. Tout d'abord, sous le coup d'un raffinement des connaissances en génétique, ce qui était perçu comme des risques aléatoires, disons de maladie, se présente maintenant de plus en plus souvent avec la certitude d'un destin (on sait que tel enfant développera telle pathologie) et du même coup ne peut plus être défini comme un risque ; par ailleurs, pour des raisons démographiques (moins de jeunes et espérance de vie plus élevée chez les personnes âgées), le partage des risques, sous la forme assurancielle, entre vieux et jeunes devient plus problématique et est susceptible d'alimenter un sérieux conflit intergénérationnel. Enfin, dans des sociétés où certaines formes d'exclusion revêtent de plus en plus un caractère de structure, ce qui était risque conjoncturel, comme le chômage, la précarité d'emploi, devient non plus un accident de parcours mais une situation qui tend à se perpétuer.

II suffit de s'y arrêter un moment pour convenir de l'importance de ces failles, en particulier la dernière. Reposant sur le principe d'une contribution monétaire des individus à l'intérieur d'un groupe, ce système assuranciel implique, dans une société salariale, qu'on soit ou qu'on ait été salarié. Le problème c'est que si on a vu par le passé s'accélérer le mouvement vers un salariat généralisé, on voit maintenant l'espace laissé au marché du travail se restreindre considérablement. Une proportion croissante de citoyens dans une société comme la nôtre n'arrive même pas à entrer sur ce marché, à s'y maintenir ou encore à s'y réinsérer. Bien plus, des proportions non négligeables n'accèdent qu'à un marché clandestin, le travail au noir sans protection sociale, ou encore ne réussissent à se maintenir qu'à la marge du marché « officiel », soit dans des emplois précaires, le plus souvent rétribués au-dessous ou au niveau du salaire minimum et, eux aussi, sans grande protection sociale. Autant de couches de la population que les régimes assuranciels n'atteignent tout simplement pas ou, au mieux, qui sont contraintes à y participer selon des modalités tendant à en réduire au minimum les bénéfices. 
Ainsi, la forme assurancielle, associée à l'instauration de l'État-providence et fondée sur le statut de travailleur salarié, peut de moins en moins remplir ses fonctions de protection. En fait, l'espace institué des solidarités sociales sous cette forme s'en trouve significativement réduit.

Mais ce n'est pas seulement par cette voie que la base assurancielle de la protection sociale et du bien-être a été laminée. Dans certains cas, pour satisfaire à des besoins nouveaux, d'importants programmes de sécurité du revenu ont plus ou moins perdu leur caractère assuranciel. Le contrat social qui à l'origine les sous-tendait a été reformulé en douce. Nous en avons des exemples au Canada et au Québec dans l'évolution du programme d'assurancechômage et, tout récemment, dans l'importante réorientation du programme fédéral de sécurité de revenu préconisée par le rapport Axworthy (1994).

Le régime d'assurance-chômage, qui date de 1941 et qui constitue l'un des programmes les plus emblématiques de l'État-providence, a été campé, on le sait, dans le cadre d'une politique visant le plein emploi et où l'assurance d'une sécurité de revenu ne devenait, en quelque sorte, qu'une mesure seconde, complémentaire. Sous la forme de cotisations en provenance d'employeurs et d'employés, l'assurance-chômage instituait sous l'égide de l'État une forme de solidarité/compromis entre travail et capital, assurant les travailleurs-cotisants contre le risque d'une perte d'emploi et permettant aussi, il ne faut pas l'oublier, de garantir le maintien du revenu de consommation indispensable à la marche de l'économie fordiste. On ne peut ici entrer dans le détail des transformations de ce régime. Qu'il suffise de signaler qu'on en est venu à. utiliser le fonds de l'assurance-chômage pour indemniser des catégories de travailleurs saisonniers (notamment les pêcheurs des côtes atlantiques), mais aussi des travailleurs engagés dans des emplois précaires dont le risque de chômage ne peut plus être considéré comme aléatoire et conjoncturel mais bien comme récurrent et structurel. Dès ce moment, l'État fédéral, à tort ou à raison, là n'est pas la question, a transformé la nature du programme et a modifié les termes du contrat entre les parties. Le régime d'assurance-chômage glisse vers la forme assistancielle. Une fraction importante des cotisants y contribuent sans en partager le risque. Ce qu'on instaure, c'est ainsi davantage la logique de la solidarité (solidarité contrainte, dirions-nous) que la logique assurancielle, pour reprendre les termes de Rosanvallon.

Première dérive qui sera suivie plus récemment par une autre : l'État central redéfinit son intervention en utilisant les fonds d'assurance-chômage non pas à de classiques prestations, mais à des mesures qui s'apparentent à la fois au workfare (les mesures d'employabilité - recyclage scolaire, stages en milieu de travail - à l'intention des assistés sociaux) et aux politiques dites actives de main-d'œuvre, principalement destinées aux chômeurs et mises de l'avant depuis les années 1970. L'O.C.D.E., on s'en souviendra, incitait alors fortement les pays développés à s'orienter vers ce genre de politique, notamment à travers le développement de la formation professionnelle continue sous la responsabilité et le contrôle renforcés de l'entreprise.

Le bilan de ces changements au niveau de l'emploi et dans la redéfinition des politiques de sécurité de revenu se résume donc ainsi : 1) une diminution de la population protégée dans le cadre d'un système de solidarité établi sur le mode assuranciel ; 2) le détournement par l'État du contrat social de type assuranciel qu'implique la mise à contribution des travailleurs et des employeurs en vue de parer au risque de se trouver sans emploi et sans ressource. Ainsi, d'importants programmes de protection sociale ont perdu leur caractère strictement assuranciel.

De là, cependant, on ne peut conclure avec Rosanvallon, pressé de nous annoncer le nouvel âge du social et du politique, que le paradigme assuranciel est actuellement en train de s'épuiser et que, du même coup, et pour cette raison, émerge un tout autre mode d'intervention de l'État dans le social.

Qu'on assiste à des réorientations dans la gestion du social, au moment où les solidarités sociales sont mises sous quasi extrême tension, est presque de l'ordre de l'inévitable. La façon, cependant, dont la question est posée par Rosanvallon réduit pour l'essentiel l'État-providence à la mise en place d'une forme assurancielle de risque contre des aléas comme la perte d'emploi, la maladie, l'accident. Elle laisse dans l'ombre, comme nous l'indiquions précédemment, certaines dimensions nécessaires pour saisir plus en profondeur la dynamique à la base de 
l'évolution de l'État-providence et, aussi, des dimensions à prendre en compte pour préciser dans quelle mouvance il est engagé. C'est ce que nous allons tenter de démontrer.

La forme assurancielle ne représente qu'une seule dimension de l'État social, dimension d'ailleurs inégalement développée selon les sociétés. Restent toutes ces mesures d'assistance qui caractérisent profondément des régimes nationaux de protection sociale comme, entre autres, l'A.F.D.C. (l'aide aux familles avec enfants dépendants) aux États-Unis, l'aide sociale au Canada et au Québec, le récent programme du Revenu minimum d'insertion (R.M.I.) en France. En fait, autour de la sécurité sociale, s'est développée une panoplie fort complexe de programmes et de mesures de nature et de portée différentes. La solidarité sociale qui s'est négociée dans l'après-guerre autour des programmes sociaux, particulièrement dans les pays à tendance sociale-démocrate, en est venue à dépasser la simple notion de protection contre les risques liés à des statuts pour rejoindre des notions plus larges de besoins et de bien-être dont la collectivité devait se rendre responsable sans condition vis-à-vis chacun de ses membres.

Quand on s'arrête à l'analyse de l'évolution de la protection sociale, on la voit effectivement osciller entre deux formes polarisées, l'assurance et l'assistance, formes qui constituent, comme nous l'avons déjà dit, quelque chose comme deux invariants dans l'évolution de l'Étatprovidence.

Cette reconnaissance phénoménologique du champ a, selon nous, d'importantes implications pour la suite de l'analyse. Ainsi, si la part de chacune des deux formes est marquée par l'histoire propre des sociétés, cette part est aussi déterminée par l'État, variable dans le temps, des rapports de forces entre groupes sociaux. Elle est aussi inégalement répartie entre la catégorie des travailleurs et des non-travailleurs. C'est même là l'axe le plus discriminant dans la répartition des risques et des protections. Le système de protection sociale dont on a hérité a en effet d'abord été construit dans le cadre précis d'une société où s'affirme la dominance des rapports salariaux, en fonction du travail salarié. L'assuré, c'est d'abord le travailleur salarié, et le mieux assuré, c'est le travailleur posté dans les meilleurs emplois. Aux non-travailleurs sont réservées des mesures d'assistance qui n'offrent pas les mêmes garanties. Et le risque auquel vient pallier à différents degrés l'assurance, c'est au sens fort le risque prolétarien : celui de se retrouver sans emploi et sans revenu dans une société marchande et salariale.

La place du paradigme assuranciel doit être vue comme l'objet d'un enjeu, et non comme l'aboutissement d'un déterminisme fonctionnel basé sur de simples changements dans la nature des risques. Le système assuranciel n'est qu'une forme de compromis/solidarité, ce n'est pas l'essence de l'État-providence.

Dans le contexte actuel de restructuration du marché du travail, de crise fiscale, d'ébranlement d'institutions intégratrices et productrices de solidarités importantes comme la famille, le quartier ou même la classe sociale d'appartenance, de revendications multiples et insistantes de groupes sociaux cherchant à faire reconnaitre leur identité et leur place dans l'ensemble de la société, tout cela mène à une exacerbation des rapports de forces qui sous-tendent les politiques sociales et fragilise les formes d'institutionnalisation des liens sociaux.

Que devient alors dans cette perspective le système des assurances tel qu'on l'a connu? Il y a fort à parier que ce système de protection persistera dans la mesure où le contrat qu'il implique (je paie, donc j'y ai droit) offre une garantie que d'autres contrats sociaux, comme ceux liés aux mesures d'aide sociale, n'offrent pas. Dans la mesure aussi où ceux qui en bénéficient disposent d'une meilleure marge de négociation.

En d'autres mots, il ne faut sans doute pas s'arrêter au seul niveau des modifications de la forme génétique des mesures de protection sociale, risque et assurance, mais aller aux sources de la dynamique sociale à l'œuvre, et des définitions collectives de l'espace donné à la solidarité sociale. Le scénario qui nous semble le plus probable actuellement, c'est moins l'évanouissement du système assuranciel que, premièrement, son épuration de fonctions (surajoutées) de redistribution plus large vers laquelle on a vu glisser, par exemple, l'assurance-chômage, pour revenir à des formes plus classiques et traditionnelles; secondairement, et presque par voie de conséquence, l'accès à ces assurances deviendra, ou au mieux demeurera, plus restreint, atteignant les catégories de travailleurs déjà plus favorisés. Il nous semble assez évident que le 
système assuranciel public (doublé de sa contrepartie privée) tend à s'intégrer, à se lover tout naturellement dans les processus accélérés de dualisation de la société. Sa capacité de garantir une large solidarité est de plus en plus restreinte et, dans le contexte où s'accentue la division sociale du travail, les assurances sociales risquent de devenir le fait d'une élite de travailleurs. Elles deviennent plus marginales, en effet, si on se place dans cette perspective, et pas parce qu'elles ont perdu toute leur efficacité ou parce qu'elles sont nécessairement en voie de disparaître.

\section{NOUVEAUX DROITS SOCIAUX, NOUVEAU CONTRAT SOCIAL ?}

Rosanvallon accorde une place centrale dans sa problématique à la remise en cause de la conception traditionnelle des droits sociaux. Cette conception traditionnelle, selon l'auteur, ne fait que reconnaître aux exclus du système de production un droit à une indemnisation et cela sans contrepartie de leur part. On a ainsi institué un «État passif-providence », un Étatprovidence réduit à une fonction d'indemnisateur. Ce mode institutionnel de gestion de la solidarité vis-à-vis les laissés pour compte, bien que marquant un pas souvent très important vers une plus grande reconnaissance du droit de tous à participer à la richesse collective, amène non seulement à sanctionner la marginalité des assistés, mais aussi, par son approche passive, à les maintenir dans leur statut de citoyens de seconde zone. Il est clair que les sociétés d'aujourd'hui ne peuvent plus fonctionner de cette manière. Le glissement de fractions de plus en plus nombreuses de la population vers le statut d'assistés, vers l'exclusion, oblige à penser à des stratégies plus actives, à une action plus vigoureuse de l'État.

Une autre raison incite à changer le mode de gestion des rapports entre l'État et les assistés, et cette raison est peut-être encore plus importante et significative aux yeux de Rosanvallon : le rapport État-prestataires, en prenant la forme d'un nouveau contrat où les personnes assistées cessent d'être perçues comme de simples récipiendaires de prestation pour être dorénavant interpellées comme des personnes responsables et des citoyens à part entière, réinvestit ceux-ci d'une certaine dignité, condition nécessaire à une sortie de la marginalité et de l'exclusion.

Dans le prolongement logique de ce nouveau droit social, Rosanvallon préconise l'émergence (ou la consolidation) d'une société de l'insertion. L'essentiel du contrat social tient d'une part clans le devoir de la société d'assurer des moyens d'insertion et de réinsertion des exclus du marché du travail et, d'autre part, dans le devoir des assistés de s'engager activement dans ce processus de retour au travail. Pour cela, Rosanvallon imagine le développement d'un nouveau secteur de l'économie, inscrit dans celui de la production des services, d'un secteur non marchand, pour éviter que les assistés qui s'y emploieraient soient placés en concurrence avec les travailleurs, les " vrais " travailleurs, déjà en place. Les prestataires, tout en demeurant prestataires, s'y adonneraient à la production de tout un ensemble de services. Les dépenses de l'Ëtat-providence deviendraient ainsi productives. Belle rencontre de l'économique et du social ! C'est presque un nouveau mode de gestion keynésien qui réapparaît. Le secteur ainsi constitué deviendrait "un espace de resocialisation et de réapprentissage professionnel » construit à partir d'un contrat qualifié de contrat emploi-solidarité. On peut se laisser aller à imaginer à partir de là une nouvelle topologie sociale où le secteur de l'insertion deviendrait une sorte de purgatoire permettant, à force de bonne volonté, de gagner certaines indulgences et de passer éventuellement au ciel du monde du travail, du vrai, et, enfin, d'accéder légitimement au paradis de la retraite...

Encore une fois, on se retrouve devant une analyse qui rejoint des problèmes et questions majeurs dans la conjoncture actuelle. On est aussi placé devant des propositions qui, à première vue, ne manquent pas d'intérêt et participent d'une vision généreuse du social. Et pourtant, cette analyse et la cohérence du projet proposé nous apparaissent extrêmement fragiles.

Reprenons d'abord l'idée de contrat social. Il faut ici carrément dire les choses. Il est notoire que, de différentes manières, on a depuis quelque temps déjà cherché dans plusieurs 
sociétés à redéfinir le contrat entre l'État et les prestataires, notamment dans le champ de l'assistance sociale. Là-dessus, Rosanvallon ne nous apprend pas grand-chose. Depuis quinze ans au moins, de nombreux analystes ont souligné l'important passage du welfare au workfare et plus récemment, dans le cas des États-Unis, au wedfare. Que nous disent-ils? En dehors de variantes et de différences dans l'appréciation de ce phénomène, ils s'entendent pour souligner qu'un des faits marquants depuis les années 1970, c'est la nouvelle posture en ce domaine de l'État-providence : il exige de façon de plus en plus impérative que les assistés sociaux se montrent " actifs ", qu'ils s'engagent dans des processus de réinsertion au marché du travail. Il ne donne plus facilement sans contrepartie. C'est justement une des dimensions majeures $\mathrm{du}$ passage du welfare au workfare. Il y a donc eu dans les programmes d'assistance destinés aux exclus du marché du travail une stratégie étatique plus directe, plus mobilisatrice. Ces mesures nouvelles sont venues rejoindre, comme nous l'avons déjà signalé, la perspective des politiques actives de main-d'œuvre mises en marche dans les années 1960 et 1970 . Ou encore elles ont pris la forme, plus large comme en France, de mesures « actives » de réintégration sociale des exclus.

Ce rapport préfigure-t-il celui que Rosanvallon voit comme un des axes d'un nouvel âge du politique et du social, c'est-à-dire, dans le cas présent, une posture de l'État garantissant un nouveau rapport d'échange avec le prestataire, un rétablissement de celui-ci dans sa dignité de citoyen, la reconnaissance de sa capacité à participer à son propre salut ? Et, surtout, une voie pouvant mener à une réinsertion dans les circuits des échanges sociaux et l'évitement de ce que Castel (1995) appelle désaffiliation?

Malheureusement, il faut bien constater que dans l'état actuel des choses, ce virage vers les politiques d'assistance, au moins dans les expériences poursuivies en Angleterre, aux ÉtatsUnis, au Canada et au Québec, s'est soldé par des interventions à tendance autoritaire de l'État, poussant les populations assistées vers des programmes d'employabilité, multipliant les opérations de contrôle et de surveillance, cherchant même par le jeu des normes d'accessibilité à restreindre l'accès à l'aide. Véritable harcèlement qu'on justifie par la crise fiscale ou, pire encore, par le recours à des jugements moraux sur le comportement des assistés, définis comme des personnes le plus souvent irresponsables et aux mœurs répréhensibles. Cela conduit, on le comprend bien, à une stigmatisation accrue des assistés, des «B.S. », comme on les désigne ici. On est loin d'un contrat vraiment libre liant prestataires et État.

En réalité, déjà dans les années récentes, se sont instituées des réponses politiques et politico-administratives à la crise structurelle qui mènent à l'exclusion, et cela sous la forme du workfare. Nous n'en sommes plus, comme le suggère Rosanvallon, à un modèle ou à une stratégie théorique, mais bien à une façon de faire, à des pratiques devenues ou tendant à devenir dominantes. Il ne s'agit pas seulement, en d'autres mots, de pratiques annonciatrices d'une nouvelle conception des droits sociaux de type contractuel, et encore moins d'un contrat libre.

Derrière ces orientations nouvelles de certaines politiques d'assistance, où on voit l'État interpeller les populations assistées et chercher à les mobiliser dans un mouvement de retour au travail, s'impose une pesanteur sociale qui trouve ses assises dans des grands intérêts économiques et dans le poids d'une bureaucratie étatique.

\section{LA SOCIÉTÉ D'INSERTION}

Peut-on contrevenir à cette pesanteur sociale ? L'expérience des dernières décennies est encore une fois peu concluante. Dans la très grande majorité des cas, nous sommes face à des constats d'échec, ici comme ailleurs. Peut-on espérer que, dans la perspective de l'émergence d'un nouveau contrat social et de l'établissement d'une société d'insertion, se présente ainsi une voie de sortie ? Tout porte à croire que non.

Il est imaginable de penser qu'on puisse arriver à convaincre les populations assistées qu'en regard des prestations reçues, elles ont des devoirs à remplir, une responsabilité vis-àvis la collectivité. On pourrait peut-être changer le discours, comme cela se fait déjà au Québec 
à travers une nouvelle publicité " positive », sur ce que sont les assistés et amener l'opinion publique et les assistés eux-mêmes à reconnaître leur dignité comme citoyens et leur capacité de réinsertion sociale et professionnelle. Cependant, un tel discours ne peut tenir longtemps lorsqu'il y a continuellement bris de contrat : ce n'est qu'une faible proportion des assistés qu'on aide à réintégrer le marché du travail et encore, quand on y parvient, c'est dans des emplois souvent précaires, de courte durée, à faible salaire et sans grande protection sociale. Ils entrent ainsi au mieux dans une espèce de zone grise située à la périphérie du marché du travail, une zone s'articulant de façon de plus en plus systématique à des programmes de bienêtre et de sécurité du revenu : passage par des stages en entreprise, payés par l'État, des programmes de formation avec allocation aussi payés par l'État à des emplois de second ordre, emplois pour lesquels l'État dans certains cas devra verser des suppléments de revenu étant donné la faiblesse des salaires; suite à cela, il y a souvent un retour au statut de chômeur ou au statut de simple assisté, puis un nouvel engagement dans des programmes d'employabilité, et ainsi de suite.

En fait, on a assisté à l'émergence d'un secteur hybride de l'économie où se rencontrent État ett petites entreprises, surtout dans le domaine des services, secteur que certains cherchent à définir comme une zone tampon. Cela apparaît en clair dans les études sur la difficile insertion professionnelle des jeunes où on voit s'instituer entre l'école et le marché du travail un système social ménageant ou aménageant une transition devenue longue, tortueuse. On comprend facilement que l'État et un certain secteur de l'économie y trouvent chacun leur compte : l'État, avec moins de chômeurs et assistés officiellement déclarés, l'entreprise, avec une réserve d'employés à bon compte. On comprend aussi que les luttes se font souvent vives entre syndicats, patrons et État, devant cette frange de main-d'œuvre peu coûteuse, facilement corvéable et devenue «flexible». Mais des luttes sont aussi menées par les regroupements d'assistés sociaux, qui y voient une exploitation des leurs, ou encore par les jeunes, comme on l'a vu récemment en France avec la vive réaction au projet d'un S.M.I.C.-jeune.

On se trouve donc devant un espace social déjà constitué et en voie de consolidation et un espace qui devient un enjeu majeur dans les processus d'exclusion/réinsertion. Rosanvallon propose qu'on développe ce secteur pour en faire un «secteur d'économie intermédiaire de l'intégration sociale », un espace de " resocialisation et de réapprentissage professionnel. » Il s'agirait non d'un secteur marchand, mais d'un secteur d'emploi-solidarité, où les exclus, tout en contribuant au mieux-être de la société, se « réhabiliteraient ». Les dépenses de l'État deviendraient ainsi productives en même temps qu'elles assureraient un mieux-être collectif : bienfait social et bienfait économique se réaliseraient en harmonie. On pense revenir à la crise des années 1930 et à la mobilisation des chômeurs dans des travaux publics...

Encore une fois, on plonge dans l'utopie et l'imprécision, la confusion même. Ces emplois devraient être créés par l'État pour éviter d'entrer en concurrence avec le secteur marchand. Mais quels seraient ces emplois non concurrentiels ? Comment les assistés y trouveraient-ils leur compte, sur le plan de l'apprentissage, sur le plan d'un sentiment d'utilité et d'affirmation d'une certaine dignité, si, comme on peut le prévoir, ces emplois concentrés dans le secteur des services risquent d'être des emplois à très faible statut et dévalorisés par la société ? On peut en effet imaginer quelle signification sociale, quel statut serait attribué à un secteur d'activités de service devenu bien visible, regroupant des personnes dans l'ensemble peu qualifiées, dépendantes de revenus de prestation sociale. La solution Rosanvallon ne conduit-elle pas à ce que justement elle veut éviter : créer un secteur qui place carrément dans la marginalité cezux qui s'y retrouvent ? Peut-on décemment offrir un tel contrat dit contrat-solidarité aux assistés sociaux et penser que ce sera pour eux l'occasion d'une "resocialisation et d'un réapprentissage professionnel »? Et même si c'était le cas, qu'adviendrait-il d'eux après, sur le « vrai » marché du travail ?

Tout se passe en somme comme si, en s'appuyant sur des évolutions récentes de l'État social, on cherchait par une seule opération sémantique à leur donner un sens autre que celui qui est le leur, faisant abstraction du poids que continuent à exercer sur ces évolutions les transformations des structures sociales et économiques et les rapports de force, qui marquent 
l'actuelle dynamique de sociétés comme la nôtre. C'est couper les pratiques sociales de leurs conditions d'émergence et de transformation.

\section{LA DERNIÈRE DES AMBIGUÏTÉS : UNE SOCIÉTÉ D'INDIVIDUS}

Dernier axe de la nouvelle problématique du social : les sujets des politiques sociales ont changé. «L'État-providence était, écrit Rosanvallon, bien organisé pour traiter les problèmes de populations relativement homogènes, de groupes et de classes si l'on veut. » (Rosanvallon,1995, p. 197.) Il doit maintenant prendre en charge des individus tous placés dans des situations particulières, singulières. On se trouve face à un jugement d'une portée considérable que l'auteur justifie à partir d'une « sorte de révolution sociologique » à l'œuvre dans nos sociétés de fin de siècle. Il entre ainsi dans la mouvance d'une analyse sociologique et philosophique de la société qui se situe près des thèses postmodernistes dont nous parlions au début et qui tendent à s'imposer de plus en plus en sociologie. Il y a ici quelque chose comme une résurgence de l'individualisme méthodologique, c'est-à-dire de cette position épistémologique qui consiste à postuler que la société n'est qu'un regroupement d'individus, au mieux de sujets. Cette position, surtout associée à un traitement quantitatif des faits sociaux (à une sociologie à la Lazarsfeld et à la Boudon), semble paradoxalement revitalisée suite à l'engouement récent pour les analyses qualitatives.

Dans la mesure où elles passent par l'observation des personnes dans leur réalité (historique), ces analyses tendent à mettre en évidence leurs particularismes, leurs différenciations. La force de persuasion de cette approche lui vient du surplus de sens qu'elle va ainsi chercher dans le vécu des individus. Mais ce recours au sujet individuel ne devient véritablement une entreprise sociologique que dans la mesure où il débusque des régularités sous les diversités, permettant d'aller un pas plus loin dans la compréhension du social. La quête sociologique se situe toujours, et pour l'essentiel, aux frontières mouvantes de l'individuel et du collectif.

Cela dit, il nous semble important de souligner le danger d'un glissement de ce qui constitue un mode d'appréhension, une épistémologie valorisée par les temps qui courent, à des jugements de fait sur l'état et la dynamique actuels du social.

Vue dans cette perspective, la population des exclus rejoint justement cette notion de masse, notion chargée idéologiquement et qui a longtemps hanté l'analyse sociologique. La société n'est plus qu'un agrégat d'individus. Il n'y a plus, répète Rosanvallon, de trajectoire collective, seulement des trajectoires individuelles. Il s'ensuit que l'État-providence devra s'orienter vers des interventions disons particularistes (au sens ordinaire, mais aussi au sens parsonnien du terme).

Un rappel rapide des caractéristiques des assistés et exclus et de leur trajectoire et, surtout, le rappel de la dynamique des rapports sociaux dans nos sociétés nous amènent à d'autres conclusions. Dès le départ, ces assistés et ces exclus ont au moins une caractéristique commune : ils sont mis hors du marché du travail, ils sont hors du coup dans une société organisée sur la base des rapports salariaux. On pourrait toujours vouloir argumenter qu'à cela près, ces exclus constituent une population relativement hétérogène. Et pourtant, les sujets de l'aide sociale demeurent dans l'ensemble encore socialement marqués. Il suffit, pour en avoir une idée, de prendre connaissance des caractéristiques des prestataires de l'A.F.D.C. (l'aide aux familles avec enfants dépendants) aux États-Unis, de la clientèle de l'aide sociale au Canada et au Québec, des caractéristiques des « remistes » en France. Il s'agit de personnes peu scolarisées dans leur grande majorité, de travailleurs potentiels sans grande qualification professionnelle, de personnes discriminées sur le marché du travail, de clientèles où, dans les faits, sont surreprésentés les jeunes et les femmes (en particulier les mères monoparentales), où sont aussi surreprésentés certains groupes ethnoculturels, qu'on pense aux noirs, aux hispanophones en territoire américain et aux immigrants. Ces prestataires se recrutent très majoritairement dans les milieux sociaux les plus désorganisés, zones grises des centres urbains, milieux ruraux en déclin, banlieues pauvres. Il est clair qu'ils ne sont pas n'importe qui. Et cela même si, dans la crise actuelle, on voit tomber dans la marginalité des catégories ou couches sociales 
appartenant traditionnellement aux classes moyennes et des proportions grandissantes de personnes aptes au travail. Ces changements sont significatifs, et il n'est pas question de les nier, bjen au contraire. Mais cela ne fait pas des assistés et des exclus un pur amas ou agrégat d'individus d'abord caractérisés par leur hétérogénéité.

Plus profondément, il faut bien reconnaître que, sous-jacents à cette composition de la population des assistés et des exclus, sont à l'œuvre des processus de reproduction des inégalités, processus qui mènent à l'exclusion et à la désaffiliation, comme l'a souligné, entre autres, Robert Castel (1995). Ces processus impliquent des trajectoires et des scénarios qui sont récurrents et dont le caractère collectif nous semble tout à fait indéniable. Il s'agit de trajectoires qui se construisent, toujours selon Castel, dans un champ social marqué par deux pôles : le degré d'intégration socio-professionnelle des individus et leur participation à des réseaux sociaux, autour de la famille, du quartier, des communautés locales. Il nous apparaît aussi que leurs positions sont liées à leurs ressources en termes de capital culturel, social et économique, et que la composition de ce capital, de même que la capacité de le mobiliser, marquent les limites de leur action et particulièrement le sens que peut prendre leur trajectoire.

La « révolution sociologique » dont parle Rosanvallon nous semble donc avoir des limites bien évidentes. Elle nous alerte cependant sur l'ampleur de certains processus en cours dans nos sociétés. Tout se passe un peu comme si nous vivions des mutations analogues à celles qui sont apparues au moment de la révolution industrielle, soit des déplacements majeurs de populations, d'un statut à l'autre, d'une position à l'autre dans des structures sociales en changement. Les frontières nationales, de classe et même de sexe se brouillent partiellement, le marché du travail se restructure, déplaçant des masses de travailleurs. Ce qui peut donner à croire qu'il n'y a plus de classes et de clivages aussi prononcés qu'auparavant selon les appartenances sociales; ce qui conduit facilement certains à mettre en évidence la fluidité comme caractíristique des sociétés contemporaines et à juger que dorénavant, le social se structure à partir de trajectoires individuelles s'exerçant dans un espace social ouvert; ce qui conduit aussi à laisser entendre que l'idéologie et la culture de l'individualisme rejoignent et recouvrent complètement la réalité sociale et sa dynamique. Pourtant, à l'évidence, des luttes collectives continuent à s'affirmer et on voit s'approfondir des divisions sociales et économiques et se creuser des inégalités de tous ordres à l'intérieur des espaces nationaux et au niveau plus large du système mondial.

Pierre DANDURAND

Département de sociologie Université de Montréal C.P. 6128, Succ. Centre-ville Montréal, (Québec), Canada H3C 3J7

\section{RÉSUMÉ}

En marge d'un ouvrage de Pierre Rosanvallon, l'auteur livre ses réflexions sur certaines des transformations actuelles de l'État-providence. Il conteste le fait que la forme assurancielle soit un mode " périmé » de régulation des solidarités ; il commente les «nouveaux droits sociaux » régissant les rapports État-prestataire; il exprime de forts doutes sur la possibilité d'implanter une « société d'insertion » et sur l'interprétation voulant que l'État-providence ait maintenant à dispenser ses services à une « société d'individus ».

\section{SUMMARY}

From the reading of a work by Pierre Rosanvallon, the author presents his reflections on certain transformations in today's welfare state. He contests the fact that insurance is an outmoded means of regulating solidarity; he comments on the "new social rights" which regulate relations between the state and beneficiaries; and he expresses grave doubts concening the possibility of setting up a "integrating society" and concerning the interpretation whereby the welfare state must now dispense its services to a "society of individuals". 


\section{RESUMEN}

A partir de un trabajo de Pierre Rosanvallon, el autor reflexiona sobre ciertas transformaciones actuales del Estado benefactor. El autor pone en duda que el modelo de la seguridad sociale sea un modo "perimido " de regulación de las solidaridades, comenta además los « nuevos derechos sociales » que rigen las relaciones Estado-beneficiario y expresa grandes dudas respecto a la posibilidad de implantar una " sociedad de inserción » y a la interpretación que pretende que el Estado benefactor tenga actualmente que dispensar servicios a una « sociedad de individuos ».

\section{BIBLIOGRAPHIE}

CASTEL, Robert (1995), Les Métamorphoses de la question sociale, Paris, Fayard.

DONZELOT, Joseph (1986), L'Invention du social, Paris, Fayard.

EWALD, François (1985) L'État-providence, Paris, Grasset.

GOUVERNEMENT DU CANADA (1994), Ministère du développement des ressources humaines, Programme : Emploi et croissance. La Sécurité sociale dans le Canada de demain (rapport Axworthy).

JENCKS, Christopher (1992), Rethinking Social Policy, Cambridge et Londres, Harvard University Press.

ROSANVALLON, Pierre (1995), La Nouvelle Question sociale. Repenser l'État-providence, Paris, Seuil. 